\title{
Selective serotonin reuptake inhibitors may lead to improved cataract surgery outcomes in patients with amblyopia
}

This article was published in the following Dove Press journal:

Clinical Ophthalmology

\section{Kevin Z Xin \\ Christina R Prescott}

Department of Ophthalmology, Johns Hopkins Wilmer Eye Institute, Baltimore, MD, USA
Correspondence: Christina R Prescott Wilmer Eye Institute, Johns Hopkins Medical Institutions, Woods Building 367, 600 North Wolfe St., Baltimore, MD 21287, USA

$\mathrm{Tel}+\mid 4108367010$

Fax + I 4108930480

Email cpresco4@jhmi.edu
Purpose: To evaluate the effects of selective serotonin reuptake inhibitor (SSRI)/serotonin norepinephrine reuptake inhibitor (SNRI) medications in combination with cataract surgery in treating amblyopia in adult patients.

Patients and methods: A retrospective chart review study was conducted on patients who had undergone cataract surgery at the Johns Hopkins Hospital Wilmer Eye Institute. Six inclusion criteria were used to assess patient eligibility: 1) $>18$ years of age, 2) diagnosis of amblyopia, 3) diagnosis of cataract and treatment with surgery, 4) electronic medical record contains pre-surgery and post-surgery visual acuity (VA) measurements, 5) electronic medical record contains information on whether the patient was ever prescribed a SSRI/SNRI and the treatment duration, and 6) interocular VA difference of two lines or more on Snellen chart prior to cataract surgery. From each record, preoperative VA, postoperative VA, date of surgery, date at which postoperative VA was measured, and age at surgery were collected. Results: A total of 237 patients were included, with 38 of them being on SSRI/SNRI. The mean improvement in VA after surgery was not significantly greater in patients on SSRI/ SNRI (SSRI/SNRI: $-0.276 \operatorname{logMAR,~control:~}-0.192 \operatorname{logMAR}, p=0.15$ ). Multivariable regression was subsequently performed and while holding all other variables constant, demonstrated a statistically significant improvement in VA in patients on SSRI/SNRI $(95 \%$ CI: $-0.194,-0.0116, p=0.03)$. The regression analysis further demonstrated that advanced age has an adverse effect on the change in post-op VA (CI: $3.34 \times 10^{-3} \operatorname{logMAR}, 9.77 \times 10^{-3}$ $\log$ MAR, $p<0.005)$. Worse baseline VA is associated with a greater improvement in post-op VA $(95 \%$ CI: $-0.659 \log$ MAR, $-0.463 \log$ MAR, $p<0.005)$ but adverse effect on the absolute post-op VA (95\% CI: $0.341 \log$ MAR, $0.544 \log$ MAR, $p<0.005)$.

Conclusion: This study suggests that patients with amblyopia undergoing cataract surgery may potentially have a greater visual improvement when treated with SSRI/SNRIs.

Keywords: amblyopia, cataract surgery, selective serotonin reuptake inhibitor (SSRI)/ selective norepinephrine reuptake inhibitor, visual plasticity, dopamine

\section{Introduction}

Amblyopia, sometimes referred to as "lazy eye", is estimated to affect $1-4 \%$ of children and is associated with physiological alterations in the visual cortex early in life. ${ }^{1,2}$ This pathology is caused by abnormal visual experiences due to binocular misalignment, image degradation, or form deprivation early in life. ${ }^{2}$ Although some studies performed in the late-1970s and early-1980s suggested that the retina was the primary site of amblyopia, the current consensus is that amblyopia originates predominantly from alterations in neural circuitries in the 
primary visual cortex. ${ }^{3-5}$ The classic work of Hubel and Wiesel (1970) on visually deprived cats demonstrated that the visual cortex is molded by experience during "critical periods" in early life. ${ }^{6,7}$ These critical periods are time windows during which cortical plasticity is enhanced and neural circuits display a heightened sensitivity to acquired instructive and adaptive signals from the external environment. ${ }^{2}$ Treatment of amblyopia is thought to be ineffective after the critical period has ended. This is why amblyopia treatments, such as atropine penalization and patch implementation in combination with correction of refractive error, are performed during childhood. ${ }^{8}$

Recent research in animal models has shown that the visual cortex of the adult brain is not fully "hardwired", as previously thought. Following specific treatments, the adult brain can acquire a certain degree of plasticity even well after the previously defined critical period. ${ }^{7}$ This has been achieved in rats by inducing the release of endogenous neuromodulators, such as norepinephrine, acetylcholine, or dopamine. ${ }^{2}$ In agreement with this, Vetencourt et al demonstrated in 2008 that chronic treatment with the selective serotonin reuptake inhibitor (SSRI) Fluoxetine reinstates ocular dominance plasticity following monocular deprivation and promotes recovery of normal visual functions in adult amblyopic animals. ${ }^{9}$ Vetencourt et al also found that chronic SSRI administration increased expression of brain-derived neurotrophic factor (BDNF) and its primary receptor, TrkB. Rats intracortically injected with exogenous BDNF via osmotic minipumps exhibited greater improvement in binocular vision than control rats not injected with BDNF. Increased BDNF activates genes regulating plasticity, allowing a functional modification of neuronal circuitries that underlie recovery from amblyopia. ${ }^{9}$

Previous studies have examined the effect of SSRIs and other neuromodulators in treating amblyopia. In 2019, Sharif et al demonstrated in a 40-patient clinical trial that the SSRI Fluoxetine may be beneficial in the management of adult and adolescent amblyopia. ${ }^{10}$ In 2010, a PEDIG (Pediatric Eye Disease Investigator Group) pilot study was initiated to evaluate the use of levodopa as a treatment for residual amblyopia. The study demonstrated temporary improvement in visual acuity (VA) that regressed after drug use ceased. ${ }^{11}$ Likewise, in 2015, Yang et al showed that older amblyopic children (ages 13-17) treated with carbidopa-levodopa had greater visual cortex activation. ${ }^{12}$ Serotonin norepinephrine reuptake inhibitors (SNRIs) work via a mechanism similar to that of SSRIs, by mixed presynaptic inhibition of both serotonin and norepinephrine uptake. ${ }^{13}$ However, no animal studies to date have demonstrated the effect of SNRIs in treating amblyopia. The SSRIs approved by the Food and Drug Administration (FDA) for treatment of depression are Citalopram, Escitalopram, Fluoxetine, Paroxetine, Sertraline, and Vilazodone. The SNRIs approved by the FDA for treatment of depression are Desevenlafaxine, Duloxetine, Levomilnacipran, and Venlafaxine.

Studies have also evaluated the efficacy of refractive correction, either by spectacle correction or refractive surgery, in treating amblyopia by improving image quality in the amblyopic eye. ${ }^{1,14,15}$ For instance, many previous studies have demonstrated that spectacle correction accompanied by additional treatments such as part-time occlusion, ${ }^{16-18}$ perceptual learning, ${ }^{19,20}$ or binocular treatment can also improve amblyopia. ${ }^{14,21-23}$ Likewise, a 2004 clinical study done at the Wilmer Eye Institute demonstrated improvements in VA after LASIK surgery in patients with amblyopia. $42.8 \%$ of patients reported postoperation improvement in best-corrected VA in their amblyopic eye while $33.3 \%$ of patients reported postoperation improvement in uncorrected VA in their amblyopic eye. ${ }^{24}$ Likewise, in 2012 deWitt demonstrated improved best-corrected VA in patients who received bilateral intraocular lens implants to treat presbyopia. ${ }^{25}$ Despite these results, the general consensus is that successful recovery of VA in amblyopic adults is diminished after the "critical period", approximately 8 years of age. ${ }^{24,26-29}$

The aforementioned studies evaluated the efficacy of neuromodulators or refractive surgery alone (without controlling for neuromodulatory medications) in treating amblyopia. The effect of using vision correcting surgery in combination with neuromodulatory medications, such as SSRIs or SNRIs, in treating amblyopia in adult populations has not been examined in a clinical study. In this study, we evaluated the effects of SSRI/SNRI medications in combination with cataract surgery in treating amblyopia in adult patients.

\section{Patients and methods}

The study consisted of patients with amblyopia who had cataract surgery along with intraocular lens placement performed at the Johns Hopkins Hospital Wilmer Eye Institute between August 1, 2012 and July 15, 2018. This retrospective record review study was approved by the Johns Hopkins Institutional Review Board. A waiver of 
the HIPAA Privacy Authorization requirement was granted by the IRB due to the logistical difficulties anticipated with obtaining consent from all patients whose records were reviewed, some of whom are no longer patients of Johns Hopkins. To ensure patient privacy, all information was de-identified and each patient was given a unique study number. The study number was only linked to the medical record number through a secure cross-walk table, which was erased after completion of the study.

A set of six criteria was used to assess patient eligibility for inclusion in this study. The inclusion criteria were: 1) $>18$ years of age, 2) diagnosis of amblyopia, 3) diagnosis of cataract and subsequent treatment with surgery, 4) patient's electronic medical record contains presurgery and post-surgery VA measurements, 5) electronic medical record contains information on whether the patient was ever prescribed a SSRI/SNRI and the treatment duration, and 6) an interocular VA difference of two lines or more on the Snellen chart prior to cataract surgery. A patient failing to meet any of the above criteria was excluded from this study.

The records of 540 patients were reviewed, and 237 patients met all inclusion criteria. Thirty-eight patients were on a SSRI/SNRI at time of surgery and for at least 2 weeks postoperatively (experimental group). A total of 199 patients were not on a SSRI/SNRI at time of surgery (control group). Data collected included preoperative VA measurement, postoperative VA measurement, date of surgery, date at which postoperative VA measurement was recorded, and age at the time of surgery. All data were obtained from the EPIC electronic medical record system used at the Wilmer Eye Institute. All VA measurements on EPIC were recorded via the Snellen system. Given the measurements in EPIC were recorded by multiple ophthalmologists over a 6-year period, it is unclear to the study team which scoring algorithms were used to obtain each of the VA measurements.

Statistical analysis was performed via Excel (Microsoft Corporation, Redmond, WA, USA) software and GraphPad Prism software (GraphPad Software Inc., La Jolla, CA, USA). For analysis purposes, all VA measurements were converted from the Snellen system to the logMAR system via the equation $\log$ MAR visual acuity $=-\log _{10}$ (Snellen visual acuity). In the $\log$ MAR system, a VA of 0 corresponds to normal 20/20 vision while a greater VA value corresponds to worse vision. Hence in this paper, a negative change in logMAR corresponds to an improvement in VA whereas a positive change in $\log$ MAR corresponds to an exacerbation in VA.

\section{Results}

The study comprised of 237 patients, divided into two groups, depending on whether they were on a SSRI/SNRI at the time of surgery and for at least 2 weeks postoperatively. The mean age of the SSRI/SNRI-group was 67.6 years and that of the control group was 69.7 years. Difference in age between the SSRI/SNRI group and control group was not statistically significant (SSRI/SNRI: 67.6 years, control: 69.7 years, $p=0.25$ ). The mean preoperative $\log$ MAR VA of the SSRI/SNRI group was 0.543 and that of the control group was 0.604. Difference in pre-op logMAR VA between the SSRI/SNRI group and control group was not statistically significant (SSRI/SNRI: 0.543, control: $0.604, p=0.31$ ) (Table 1).

Improvement in VA after surgery was $-0.276 \log \mathrm{MAR}$ for the SSRI/SNRI group and $-0.192 \log$ MAR for the control group. This difference is not statistically significant (SSRI/SNRI: $-0.276 \log$ MAR, control: $-0.192 \log$ MAR, $p=0.15)$. A multivariable regression analysis was then performed by creating a model that regressed postoperative improvement in VA on predictor variables, including baseline VA, age at the time of surgery, time between surgery and post-op VA measurement, and whether the patient was on a SSRI/SNRI. Our model demonstrated that, holding all the other characteristics constant, patients in the SSRI/SNRI group had a $-0.103 \operatorname{logMAR}$ greater improvement in post-op VA compared to the control group. Based on the regression analysis model, the effect SSRI/SNRI treatment had on post-op VA is statistically significant (95\% CI: $-0.194,-0.0116, p=0.03$ ) (Figure 1).

The multivariable regression analysis further demonstrated that baseline VA as well as age at time of surgery have a significant effect on the improvement in postoperative VA. As baseline VA worsens by $1 \log$ MAR, the improvement in post-op VA increases by $-0.561 \log \mathrm{MAR}$ (95\% CI: $-0.659 \log$ MAR, $-0.463 \log \mathrm{MAR}, p<0.005$ ). However, an additional regression analysis was performed, examining the effect of baseline VA on absolute post-op VA. This regression demonstrated that as baseline VA worsens by $1 \log M A R$, the improvement in post-op VA decreases by $0.443 \log$ MAR (95\% CI: $0.341 \log$ MAR, $0.544 \log \mathrm{MAR}, p<0.005)$. As age at the time of surgery increases by 1 year, the improvement in post-op VA decreases by $6.55 \times 10^{-3} \log$ MAR (CI: $3.34 \times 10^{-3} \log$ MAR, $9.77 \times 10^{-3} \log$ MAR, $\left.p<0.005\right)$. Lastly, the multivariable regression demonstrated that time duration between surgery and postoperative VA measurement does not have a significant impact on postoperative improvement in VA (95\% CI: 
Table I Pre- and postoperative characteristics for patients in SSRI/SNRI and control group

\begin{tabular}{|c|c|c|}
\hline Characteristic & SSRI/SNRI group & Control group \\
\hline Age at surgery (years $\pm S D$ ) & $67.6 \pm 7.6$ & $69.7 \pm 10.8$ \\
\hline Pre-op VA (logMAR \pm SD) & $0.543 \pm 0.286$ & $0.604 \pm 0.351$ \\
\hline Post-op VA (logMAR \pm SD) & $0.266 \pm 0.190$ & $0.4 I I \pm 0.322$ \\
\hline Time between surgery and post-op VA measurement (years \pm SD) & $1.79 \pm 1.12$ & $1.51 \pm 1.32$ \\
\hline Males, $n(\%)$ & $10(26)$ & $79(40)$ \\
\hline Females, $\mathrm{n}(\%)$ & $28(74)$ & $120(60)$ \\
\hline
\end{tabular}

Abbreviations: SSRI, selective serotonin reuptake inhibitor; SNRI, serotonin norepinephrine reuptake inhibitor; VA, visual acuity.

$\left.-8.70 \times 10^{-5} \log \mathrm{MAR}, \quad 5.54 \times 10^{-5} \log \mathrm{MAR}, \quad p=0.66\right)$. Moreover, the difference in time between when the postoperative VA measurements were performed in the SSRI/ SNRI group and control group was not statistically significant (SSRI/SNRI: 1.79 years, control: 1.51 years, $p=0.21$ ) (Table 2).

\section{Discussion}

It has been long observed that amblyopia treatment is most effective within the critical period of visual development, usually defined as up to 8 years of age. If left untreated past this critical period of cortical brain plasticity, amblyopia can result in permanently impaired vision. ${ }^{11}$ Recently, preliminary studies using adult animal models have demonstrated the ability of SSRIs to extend the period of

Improvement in visual acuity prior to multivariable regression

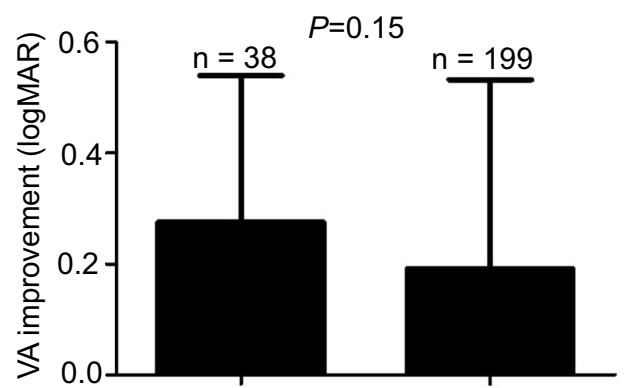

Improvement in visual acuity after multivariable regression

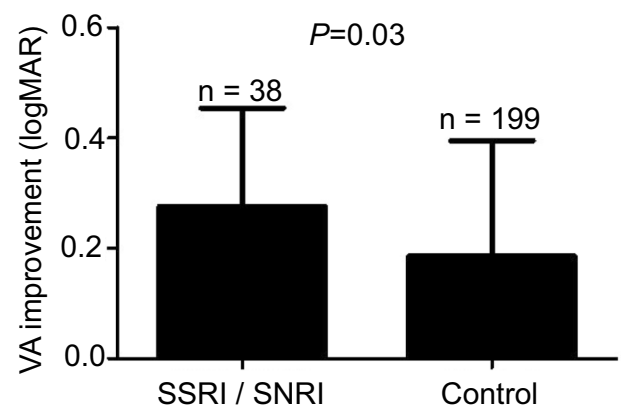

Figure I Graph of improvement in visual acuity post-op. plasticity in amblyopic eyes. However, whether this same effect occurs in adult humans after refractive surgery is unknown; therefore, we decided to perform this retrospective clinical chart review study.

Our study demonstrated that SSRI/SNRI administration at the time of cataract surgery may lead to an improvement in postoperative VA after all other contributing variables are held constant. To the best of our knowledge, there has only been one other large study looking at the effect of SSRI/SNRI administration on amblyopia treatment. This 42-patient study, performed in 2017, demonstrated that patients receiving fluoxetine along with computerized perceptual training and eye patching did not have significantly better improvements in VA when compared to control group patients receiving only computerized perceptual training and eye patching. ${ }^{30}$ In addition, two smaller studies have examined the effects of SSRI/SNRI administration on brain plasticity and demonstrated no significant difference. First, a 2016 clinical study showed that $20 \mathrm{mg} /$ day of Fluoxetine for 19 days did not significantly affect visual perceptual learning in humans. ${ }^{31}$ Second, a 2014 clinical study consisting of 7 patients with amblyopia demonstrated that administration of $20 \mathrm{mg}$ Citalopram for 2 weeks did not lead to a statistically significant improvement in VA. ${ }^{32}$

The results of our regression analysis suggest that SSRI/ SNRI treatment in combination with surgical intervention may lead to a significant improvement in VA. The difference between the results of our study and those of prior studies may be because we looked at the effect of SSRI/SNRI combined with a surgical intervention to improve visual input to the amblyopic eye, rather than the effect of SSRI/ SNRI alone or effect of SSRI/SNRI coupled with non-surgical treatment options. Another difference was that the patients in the prior studies were treated for 4 or fewer weeks, whereas in our study the average duration of treatment with an SSRI/SNRI was 101 weeks (1.9 years). 
Table 2 Multivariable regression model for factors affecting postoperative visual acuity

\begin{tabular}{|l|l|l|l|}
\hline Parameter & $\boldsymbol{\beta}^{*}$ & $\mathbf{9 5 \%} \mathbf{C l}$ & $\boldsymbol{p}$ \\
\hline Time between surgery and postoperative VA measurement (years) & $-1.60 \times 10^{-5}$ & $\left(-8.70 \times 10^{-5}, 5.54 \times 10^{-5}\right)$ & 0.66 \\
Age at surgery & $6.55 \times 10^{-3}$ & $\left(3.34 \times 10^{-3}, 9.77 \times 10^{-3}\right)$ & $<0.005$ \\
Baseline VA & -0.561 & $(-0.659 .-0.463)$ & $<0.005$ \\
SSRI/SNRI & -0.103 & $(-0.194,-0.0116)$ & 0.03 \\
\hline
\end{tabular}

Note: $* \beta$ is the estimated beta-coefficient of the linear regression model.

Abbreviations: SSRI, selective serotonin reuptake inhibitor; SNRI, serotonin norepinephrine reuptake inhibitor; VA, visual acuity.

In addition to the primary finding that patients on SSRI/ SNRIs may have a greater improvement in vision after cataract surgery, our study demonstrated that younger patients and patients with poorer initial VA had greater improvements in postoperative VA. These findings are consistent with trends noted in prior studies, and support the validity of our analysis. A 2000 study performed in the UK demonstrated that patients ages 60-69 were 4.6 times more likely to achieve a VA of $6 / 12$ or better postoperatively compared with patients ages $80+{ }^{33}$ Similarly, a 2016 study performed in People's Republic of China demonstrated that increased age correlates with poorer postoperative VA. ${ }^{34}$

Worse preoperative VA is associated with a greater percent improvement in postoperative VA; however, this may be attributable to a mathematical artifact. There is more potential for VA improvement in patients with poor preoperative VA, compared with patients with less initial visual impairment. A similar trend regarding percent improvement in postoperative VA can be seen in a JAMA Ophthalmology article, which recorded the pre- and postoperative VA of patients undergoing cataract surgery in four countries: USA, Canada, Denmark, and Spain. ${ }^{35}$ In addition, the JAMA Ophthalmology article noted that with respect to absolute VA, a poor preoperative VA is associated with a worse postoperative VA. ${ }^{35}$ This is also consistent with the trend observed in our study.

There are several limitations to this study; primarily that it is a retrospective chart review, and we were not able to control the dosage or duration of medication the patients received. Given the older age of the patients in the study, the diagnosis of amblyopia is most often based on history, and the primary cause of the amblyopia likely varies between patients. Additionally, there are multiple types of amblyopia, and it is likely that refractive amblyopia will respond more favorably to cataract surgery than amblyopia secondary to ocular misalignment or other etiologies. Given the suggestive findings of this study, consideration should be given to the implementation of a clinical trial to further define the effect of SSRI/SNRIs on visual plasticity and to optimize potential treatment protocols.

\section{Conclusion}

The finding that patients with amblyopia undergoing cataract surgery may have a greater visual improvement when treated with SSRI/SNRIs gives new hope to adult patients suffering from amblyopia.

\section{Disclosure}

The authors report no conflicts of interest in this work.

\section{References}

1. Simmons K. Amblyopia characterization, treatment, and prophylaxis. Surv Ophthalmol. 2005;50:123-166. doi:10.1016/j. survophthal.2004.12.005

2. Bonaccorsi J, Berardi N, Sale A. Treatment of amblyopia in the adult: insights from a new rodent model of visual perceptual learning. Front Neural Circuits. 2014;8:82. doi:10.3389/fncir.2014.00082

3. Ikeda H, Tremain KE. Amblyopia occurs in retinal ganglion cells in cats reared with convergent squint without alternating fixation. Exp Brain Res. 1979;35:559-582.

4. Arden GB, Vaegan HCR, Powell DJ, Carter RM. Pattern ERGs are abnormal in many amblyopes. Trans Ophthlamol Soc $U K$. 1980;100:453-460.

5. Barrett BT, Bradley A, McGraw PV. Understanding the neural basis of amblyopia. Neuroscientist. 2004;10:106-107. doi:10.1177/ 1073858403262153

6. Hubel DH, Wiesel TN. The period of susceptibility to the physiological effects of unilateral eye closure in kittens. $J$ Physiol. 1970;206:419-436. doi:10.1113/jphysiol.1970.sp009022

7. Bavelier D, Levi DM, Li RW, Dan Y, Hensch TK. Removing brakes on adult brain plasticity: from molecular to behavioral interventions. J Neurosci. 2010;30:14964-14971. doi:10.1523/JNEUROSCI.481210.2010

8. Venter JA, Pelouskova M, Schallhorn SC, Collins BM. Visual acuity improvement in adult amblyopic eyes with an iris-fixated phakic intraocular lens: long-term results. $J$ Cataract Refrac Surg. 2015;41:541-547. doi:10.1016/j.jcrs.2014.06.037

9. Maya Vetencourt JF, Sale A, Viegi A, et al. The antidepressant fluoxetine restores plasticity in the adult visual cortex. Science. 2008;320:385-388. doi:10.1126/science. 1150516

10. Sharif MH, Talebnejad MR, Rastegar K, Khalili MR, Nowroozzadeh $\mathrm{MH}$. Oral fluoxetine in the management of amblyopic patients aged between 10 and 40 years old: a randomized clinical trial. Eye. 2019. doi:10.1038/s41433-019-0360-z

11. Gore C, Wu C. Medical therapies of amblyopia: translational research to expand our treatment armamentarium. Semin Ophthalmol. 2016;31:155-158. doi:10.3109/08820538.2015.1114851

12. Yang CI, Yang ML, Huang JC, et al. Functional MRI of amblyopia before and after levodopa. Neurosci Lett. 2003;339:49-52. doi:10.1016/s0304-3940(02)01465-9 
13. Lambert O, Bourin M. SNRIs: mechanism of action and clinical features. Expert Rev Neurother. 2002;2:849-858. doi:10.1586/ 14737175.2.6.849

14. Gao TY, Anstice N, Babu RJ, et al. on behalf of the Binocular Treatment of Amblyopia Using Videogames (BRAVO) study team. Optical treatment of amblyopia in older children and adults is essential prior to enrollment in a clinical trial. Ophthalmic Physiol Opt. 2018;38:129-143. doi:10.1111/opo.12437

15. Kraus CL, Culican SM. New advances in amblyopia therapy II: refractive therapies. $\mathrm{Br} \quad J$ Ophthalmol. 2018;102:1611-1614. doi:10.1136/bjophthalmol-2018-312173

16. Simmers AJ, Gray LS. Improvement of visual function in an adult amblyope. Optom Vis Sci. 1999;76:82-87. doi:10.1097/00006324199902000-00014

17. Kishimoto F, Fujii C, Shira Y, Hasebe K, Hamasaki I, Ohtsuki H. Outcome of conventional treatment for adult amblyopia. Jpn J Ophthalmol. 2014;58:26-32. doi:10.1007/s10384-013-0279-z

18. Tailor V, Bossi M, Greenwood JA, Dahlmann-Noor A. Childhood amblyopia: current management and new trends. $\mathrm{Br}$ Med Bull. 2016;119:75-86. doi:10.1093/bmb/ldw030

19. Megbelayin E, Ekpenyong SM, Azunobi J, Ejiro G. Reversal of myopic anisometropic amblyopia with occlusion therapy in a 25 year old. Ghana Med J. 2014;48:54-56. doi:10.4314/gmj.v48i1.10

20. Astle AT, Webb BS, McGraw PV. Can perceptual learning be used to treat amblyopia beyond the critical period of visual development? Ophthalmic Physiol Opt. 2011;31:564-573. doi:10.1111/j.14751313.2011.00873.x

21. Levi DM, Li RW. Perceptual learning as a potential treatment for amblyopia: a mini-review. Vision Res. 2009;49:2535-2549. doi:10.1016/j.visres.2009.02.010

22. Vedamurthy I, Nahum M, Huang SJ, et al. A dichoptic custom-made action video game as a treatment for adult amblyopia. Vision Res. 2015;114:173-187. doi:10.1016/j.visres.2015.04.008

23. Hess RF, Thompson B, Baker DH. Binocular vision in amblyopia: structure, suppression and plasticity. Ophthalmic Physiol Opt. 2014;34:146-162. doi:10.1111/opo.12123

24. Sakatani K, Jabbur NS, O'Brien TP. Improvement in best corrected visual acuity in amblyopia adult eyes after laser in situ keratomileusis. J Cataract Refract Surg. 2004;30:2517-2521. doi:10.1016/j. jers.2004.06.026
25. de Wit DW, Diaz JM, Moore TC, Moore JE. Refractive lens exchange for a multifocal intraocular lens with a surface-embedded near section in mild to moderate anisometropic amblyopic patients. $J$ Cataract Refract Surg. 2012;38:1796-1801. doi:10.1016/j. jcrs.2012.06.046

26. Holmes JM, Lazar EL, Melia BM, et al. Weise KK for the pediatric eye disease investigator group. Effect of age on response to amblyopia treatment in children. Arch Ophthalmol. 2011;129:1451-1457. doi:10.1001/archophthalmol.2011.179

27. Fronius M, Cirina L, Ackermann H, Kohnen T, Diehl CM. Efficiency of electronically monitored amblyopia treatment between 5 and 16 years of age: new insight into declining susceptibility of the visual system. Vision Res. 2014;103:11-19. doi:10.1016/j.visres.2014.07.018

28. Daw NW. Critical periods and amblyopia. Arch Ophthalmol. 1998;116:502-505.

29. Hooks BM, Chen C. Critical periods in the visual system: changing views for a model of experience-dependent plasticity. Neuron. 2007;56:312-326. doi:10.1016/j.neuron.2007.10.003

30. Huttunen HJ, Palva JM, Lindberg L, et al. Fluoxetine does not enhance the effect of perceptual learning on visual function in adults with amblyopia. Sci Rep. 2018;8:12830. doi:10.1038/s41598-01831169-z

31. Lagas AK, Black JM, Byblow WD, et al. Fluoxetine does not enhance visual perceptual learning and triazolam specifically impairs learning transfer. Front Hum Neurosci. 2016;10:532. doi:10.3389/ fnhum.2016.00532

32. Lagas AK, Black JM, Russell BR, Kydd RR, Thompson B. The effect of combined patching and citalopram on visual acuity in adults with amblyopia: a randomized, crossover, placebo-controlled trial. Neural Plast. 2019. doi:10.1155/2019/5857243

33. Wescott MC, Tuft SJ, Minassian DC. Effect of age on visual outcome following cataract extraction. Br J Ophthalmol. 2000;84:1380-1382. doi:10.1136/bjo.84.12.1380

34. Li X, Cao X, Hou X, Bao Y. The correlation of age and postoperative visual acuity for age-related cataract. Biomed Res Int. 2016;2016:7147543.

35. Norregaard JC, Hindsberger C, Alonso J, et al. Visual outcomes of cataract surgery in the United States, Canada, Denmark, and Spain. Report from the International Cataract Surgery Outcomes study. Arch Ophthalmol. 1998;116:1095-1100.
Clinical Ophthalmology

\section{Publish your work in this journal}

Clinical Ophthalmology is an international, peer-reviewed journal covering all subspecialties within ophthalmology. Key topics include: Optometry; Visual science; Pharmacology and drug therapy in eye diseases; Basic Sciences; Primary and Secondary eye care; Patient Safety and Quality of Care Improvements. This journal is indexed on PubMed

\section{Dovepress}

Central and CAS, and is the official journal of The Society of Clinical Ophthalmology (SCO). The manuscript management system is completely online and includes a very quick and fair peer-review system, which is all easy to use. Visit http://www.dovepress.com/ testimonials.php to read real quotes from published authors. 